\title{
Infinite paths with bounded or recurrent partial
}

\author{
sums
}

\author{
Lorna Booth and Ronald Meester
}

January 26, 2000

\begin{abstract}
Department of Mathematics, University of Utrecht, PO Box 80010, 3508 TA Utrecht, The Netherlands. booth@math.uu.nl meester@math.uu.nl
\end{abstract}

\begin{abstract}
We consider problems of the following type. Assign independently to each vertex of the square lattice the value +1 , with probability $p$, or -1 , with probability $1-p$. We ask whether an infinite path $\pi$ exists, with the property that the partial sums of the \pm 1 s along $\pi$ are uniformly bounded, and whether there exists an infinite path $\pi$ with the property that the partial sums along $\pi$ are equal to zero infinitely often. The answers to these question depend on the type of path one allows, the value of $p$ and the uniform bound specified. We show that phase transitions occur for these phenomena. Moreover, we make a surprising connection between the problem of finding a path to infinity (not necessarily self-avoiding, but visiting each vertex at most finitely many times) with a given bound on the partial sums, and the classical Boolean model with squares around the points of a Poisson
\end{abstract}


process in the plane. For the recurrence problem, we also show that the probability of finding such a path is monotone in $p$, for $p \geq \frac{1}{2}$.

\section{Introduction}

In this paper we consider a connected, infinite, locally-finite graph, $G$, with vertex set $V$, and edge set $E$. This will generally be $\mathbb{Z}^{2}$, although many of our results can be extended to other graphs. One particular vertex is distinguished and called the origin, 0 . To every vertex $v \in V$, we assign a random variable $X_{v}$, which takes value 1 with probability $p$ and value -1 otherwise, independently of the values at other vertices.

A path from a vertex $z_{0}$ say, is a sequence of vertices $\pi=\left(z_{0}, z_{1}, z_{2}, \ldots\right)$ such that $\left(z_{i-1}, z_{i}\right) \in E$ for $i=1,2, \ldots$ For such a path we define the partial sums $S_{n}^{\pi}=\sum_{i=1}^{n} X_{z_{i}}$ for $n=1,2, \ldots$, where, for our later convenience, we do not count the value at the starting point, $z_{0}$. We are concerned with the question of whether there exists an infinite path $\pi$ with either $\sup _{n}\left\{\left|S_{n}^{\pi}\right|<\infty\right\}$ or with $S_{n}^{\pi}=0$, for infinitely many $n$. Note that these problems are symmetric in $p$ around $\frac{1}{2}$. The answers to these questions will depend upon the types of paths we allow ourselves to use. We will consider three different types of infinite paths:

Definition On the square lattice, $\mathbb{Z}^{2}$, an oriented path, $\pi=\left(z_{0}, z_{1}, \ldots\right)$, is a path such that $z_{i}-z_{i-1}=e_{1}$ or $z_{i}-z_{i-1}=e_{2}$, for $i=1,2, \ldots$, where $e_{1}$ and $e_{2}$ are the first and second coordinate vectors. A self-avoiding path is a path whose vertices are all distinct. Finally, a just-visiting path is a path in which any vertex appears only finitely many times.

All oriented paths are self-avoiding, and all self-avoiding paths are justvisiting. Note also that the existence of an infinite self-avoiding path with 
bounded partial sums starting at some vertex, implies that there exists one from every other vertex. Such a path can can be constructed, for example, by taking the shortest self-avoiding path from the chosen vertex to the bounded path and thereafter following it. This also true for just-visiting paths, analogously.

On the integer line (where integers are connected by an edge if their difference is one), the answers to these questions for self-avoiding paths are well known, as they refer to the simple random walk. (For information about this see for example [8].) Here we have that when $p=\frac{1}{2}$ we have no bounded partial sums, but we do have partial sums that are zero infinitely often, almost surely. At all other values of $p$ we have neither behaviour. If we consider just-visiting paths, it can be shown that there are no paths with bounded partial sums, almost surely, for any value of $p$, but for all $p \in(0,1)$ there are just-visiting paths with partial sums that are zero infinitely often.

Benjamini and Peres [5], answered both questions (and many other more general ones), for self-avoiding paths on trees. Given a tree, $T$, the boundary, $\partial T$ is the set of rays or infinite self-avoiding paths emanating from the root, 0 . If we denote by $\operatorname{dim}(\partial T)$ the Hausdorff dimension of this boundary (see [7] for an explanation of Hausdorff dimension) then their theorem states that a path from 0 with bounded partial sums exists with positive probability if and only if $\operatorname{dim}(\partial T)>\log \left(\frac{1}{2 \sqrt{p(1-p)}}\right)$. If we denote the packing dimension by $\operatorname{Pdim}(\partial T)$ (see [7]), then they show that for $\operatorname{Pdim}(\partial T)<\log \left(\frac{1}{2 \sqrt{p(1-p)}}\right)$ there are no self-avoiding paths with partial sums returning to zero infinitely often, almost surely, while for $\operatorname{dim}(\partial T)>\log \left(\frac{1}{2 \sqrt{p(1-p)}}\right)$ these exist with positive probability.

A sufficient condition for having infinite paths with both bounded partial sums and partial sums that are zero infinitely often, is the existence of 
$\mathrm{AB}$ percolation, for appropriate parameter values. From our viewpoint $\mathrm{AB}$ percolation asks for the almost sure existence of a self-avoiding path of the appropriate type, which has alternate $1 \mathrm{~s}$ and $-1 \mathrm{~s}$. Thus the partial sums are zero at every other point along the path, and never exit either of the intervals $[0,1]$ or $[-1,0]$. It has been shown (for example) that AB percolation occurs on the triangular lattice for an interval of values of $p$ around $1 / 2$, see [2]. AB percolation has been shown not to occur, for any value of $p$, on the square and hexagonal lattices, see [3]. We note that $\mathrm{AB}$ percolation occurs for self-avoiding paths if and only if it occurs for just-visiting paths. An interesting feature of $\mathrm{AB}$ percolation is that its probability is not monotonic in $p$ on $[1 / 2,1]$ for many graphs, see [12].

We end this section with some notation used throughout. The product measure described above is denoted by $\mathbb{P}_{p}$. Denote by $\mathbb{E}_{p}$ the corresponding expectation operator. We call two vertices adjacent if there is an edge between them, and we call two edges adjacent if they share a vertex. We define the distance between two points, $v^{1}=\left(v_{1}^{1}, v_{2}^{1}\right), v^{2}=\left(v_{1}^{2}, v_{2}^{2}\right) \in \mathbb{R}^{2}$ as $\left|v_{1}^{1}-v_{1}^{2}\right|+\left|v_{2}^{1}-v_{2}^{2}\right|$, the $L_{1}$-distance. An interval in $\mathbb{R}$ is said to have size $s$ if it contains exactly $s$ integers.

The next section in this paper contains our principal results, and the subsequent sections contain the proofs.

\section{Principal results}

Our first theorem states that there is a non-trivial phase transition for oriented paths. 
Theorem 1 On the square lattice the following hold.

(a) There are no infinite oriented paths with sums bounded in an interval of

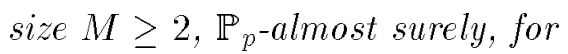

$p \in\left[0, \frac{1-\sqrt{1-1 / 4 \cos ^{2}(\pi /(M+1))}}{2}\right) \cup\left(\frac{1+\sqrt{1-1 / 4 \cos ^{2}(\pi /(M+1))}}{2}, 1\right]$

In particular, for $p \in\left[0, \frac{1}{2}-\frac{1}{2} \sqrt{\frac{3}{4}}\right) \cup\left(\frac{1}{2}+\frac{1}{2} \sqrt{\frac{3}{4}}, 1\right]$, there are no paths with sums bounded in any interval, $\mathbb{P}_{p^{-}}$almost surely.

(b) There are, $\mathbb{P}_{p^{-}}$-almost surely, infinite oriented paths with partial sums that return to zero every 42 steps for $p \in(0.475,0.525)$. This immediately implies that there are oriented paths with partial sums that are bounded and that return to zero infinitely often, with positive probability, and thus also self-avoiding and just-visiting paths with the same properties.

We see here a contrast with $\mathrm{AB}$ percolation, which occurs for no values of $p$ on this lattice.

We need a separate statement to rule out the possibility of infinite selfavoiding paths with bounded partial sums, or with partial sums that return to 0 infinitely often, for $p$ close to 0 and 1 . We shall formulate the next result for more general graphs.

For a graph $G$, let $\sigma_{G}(n)$ be the number of self avoiding walks from the origin of length $n$. Let $c_{G}=\lim _{n \rightarrow \infty} \sigma_{G}(n)^{1 / n}$, if this exists. $c_{G}$ is called the connectivity constant of $G$. For the integer lattice (and many others), existence of the connectivity constant follows from subadditivity.

Theorem 2 Let $G$ be a graph with a well-defined connectivity constant $c_{G}$.

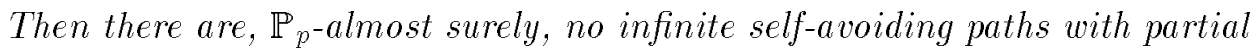
sums that return to 0 infinitely often or with partial sums that are bounded 
in some interval for

$$
p \in\left[0, \frac{1-\sqrt{1-\frac{1}{c_{G}^{2}}}}{2}\right) \cup\left(\frac{1+\sqrt{1-\frac{1}{c_{G}^{2}}}}{2}, 1\right] .
$$

For the square lattice it has been shown rigorously that $c_{\mathbb{Z}^{2}} \leq 2.7$ (see [1]), which implies that we have no infinite self-avoiding path with bounded partial sums for $p \in[0,0.035) \cup(0.965,1]$.

For just-visiting paths, the situation is quite different. For every $p \in$ $(0,1)$ we can define a minimal interval size, $I(p)$ say, to be the minimal integer, such that there exists a just-visiting path from the origin, with partial sums bounded in an interval of length $I(p)$, with positive probability.

Theorem 3 On the square lattice the following hold.

(a) For any $p \in(0,1)$ we have $2 \leq I(p)<\infty$. That is, for any $p \in(0,1)$ there is, with positive $\mathbb{P}_{p}$ probability, an infinite just-visiting path from the origin with bounded partial sums. We can take this path so that the partial sums are equal to 0 infinitely often.

(b) We have

$$
\lim _{p \rightarrow 1} I(p)=\infty .
$$

That is, for any interval $J$ on the real line we can find $p$, close to 1 , such that for this value of $p$, no just-visiting paths with all partial sums in $J$ exists, $\mathbb{P}_{p}$-almost surely.

Our next result gives a connection between two apparently unrelated processes, the percolation of just-visiting paths with bounded sums, and the classical Boolean model.

Consider a Poisson process of rate $\lambda$ in the plane and centre a diamond (with fixed orientation) of radius $1 / 2$ at every Poisson point, that is, $v$ 
is in the diamond centred at a Poisson point $x$ if the distance between $v$ and $x$ is at most $1 / 2$. This process is known to have a critical point for percolation $\lambda_{c}$, such that for $\lambda \leq \lambda_{c}$ there is no percolation (that is, the union of all diamonds contains no unbounded connected component almost surely), while for $\lambda>\lambda_{c}$ percolation occurs. Later we shall need to talk about diamond processes formed by placing a diamond of radius $r$ at each of the points of the Poisson process, and we denote the critical point for this model by $\lambda_{c}(r)$. It is can be seen by a simple scaling argument that,

$$
r_{1}^{2} \lambda_{c}\left(r_{1}\right)=r_{2}^{2} \lambda_{c}\left(r_{2}\right)
$$

for any two values, $r_{1}$ and $r_{2}$. See [13] for information on this type of model, and the results mentioned above.

Theorem 4 Consider a sequence $q_{1}, q_{2}, q_{3}, \ldots$ of numbers in $(0,1)$ converging to 1 .

(a) If $\lim \sup _{N \rightarrow \infty} 2 N^{2}\left(1-q_{N}\right)^{2}<\lambda_{c}$ then for $N$ sufficiently large there $\mathbb{P}_{q_{N}}$-almost surely does not exist an infinite just-visiting path with partial sums bounded in $[0, N]$.

(b) If either

$$
\lambda_{c}<\liminf _{N \rightarrow \infty} 2 N^{2}\left(1-q_{N}\right)^{2} \leq \limsup _{N \rightarrow \infty} 2 N^{2}\left(1-q_{N}\right)^{2}<\infty
$$

or

$$
\liminf _{N \rightarrow \infty} 2 N^{2}\left(1-q_{N}\right)^{2}=\infty,
$$

then for $N$ sufficiently large there exists with positive $\mathbb{P}_{q_{N}}$ probability an infinite just-visiting path with partial sums bounded in $[0, N]$.

Two remarks are appropriate here. In the first place, we could replace the interval $[0, N]$ in this theorem by any sequence of intervals $J_{1}, J_{2}, \ldots$, with 
$J_{k}$ of size $k$. We chose the above formulation for the sake of simplicity of the statements. Secondly, the results in (a) and (b) together will imply that we can strengthen the result of Theorem $3(\mathrm{~b})$ in the following sense:

Corollary 5 The interval size I $(p)$ defined before Theorem 3 satisfies

$$
\lim _{p \rightarrow 1}(1-p) I(p) \rightarrow \sqrt{\frac{\lambda_{c}}{2}} .
$$

Our next result gives a relationship between paths with partial sums that return to zero infinitely often and those with partial sums that do not converge to $+\infty$. It is motivated by the question of whether the probability of an infinite self-avoiding path with bounded partial sums is monotone in $p>1 / 2$. This we do not know, but we can prove a monotonicity statement for the probability of having an infinite self-avoiding path with partial sums equal to zero infinitely often. This quickly follows from the next result.

Theorem 6 On the square lattice, for any value of $p>1 / 2$ such that with positive probability, there is an infinite self-avoiding path with partial sums that do not converge to $+\infty$, there is, with positive probability, an infinite self-avoiding path with partial sums that return to zero infinitely often.

Corollary 7 On the square lattice, for $p>1 / 2$, the probability that there exists an infinite self-avoiding path with partial sums that are equal to zero infinitely often is monotone decreasing in $p$.

\section{Proof of Theorem 1}

The first part of the theorem is proved via a recurrence method, the second part with a second moment method. 
Proof of Theorem 1(a) We will show that the expected number of oriented paths of length $n$, from any point on the line $x+y=0$ to a given point $(a, b)$ (with $a+b=n, a, b \in \mathbb{Z}$ ), with sums bounded in the interval, tends to zero as $n$ tends to infinity. To see that this suffices, observe that for any interval,

$$
\begin{aligned}
& \mathbb{E}_{p}(\text { number of paths from the origin of length } n \text { with } \\
& \text { partial sums in the interval) }= \\
& \mathbb{E}_{p} \text { (number of paths to the origin of length } n \text { with } \\
& \text { partial sums in the interval })= \\
& \mathbb{E}_{p} \text { (number of paths to the point }(a, n-a) \text { from the line } \\
& x+y=0 \text { with partial sums in the interval }),
\end{aligned}
$$

for any $a \in \mathbb{Z}$.

Given an interval, $I$, of size $M$, and a point $(a, b)$ (with $a+b>0)$ define $V_{(a, b)}$ to be the random vector with elements $v_{(a, b), i}, i \in I$ which record the number of oriented paths from $x+y=0$ to $(a, b)$ which have partial sums bounded in the interval and final partial sum $i$.

Let $\Lambda_{(a, b)}$ be the random $M \times M$ matrix that has entries,

$$
\lambda_{i j}= \begin{cases}1 & i=j-1 \\ 0 & \text { otherwise }\end{cases}
$$

if $(a, b)$ is assigned value +1 , and

$$
\lambda_{i j}=\left\{\begin{array}{cc}
1 & i=j+1 \\
0 & \text { otherwise }
\end{array}\right.
$$

if $(a, b)$ is assigned value -1 . 
Then,

$$
V_{(a, b)}=\Lambda_{(a, b)}\left(V_{(a-1, b)}+V_{(a, b-1)}\right)
$$

We now take the expectation of both sides of this equation, noting two things. The first is that $\Lambda_{(a, b)}$ is independent of $V_{(a-1, b)}$ and $V_{(a, b-1)}$. The second is that the expectation of $V_{(a, b)}$ is the same as that of $V_{(c, d)}$ if $a+b=$ $c+d$. Thus we may write $\bar{V}_{n}$ to be the expectation of any $V_{(a, b)}$ with $a+b=n$. Let $\bar{\Lambda}$ be the expectation of $\Lambda_{(0,0)}$ (which is also the expectation of $\Lambda_{(a, b)}$ for any $(a, b)$, as they are identically distributed). Hence we have that,

$$
\begin{aligned}
\bar{V}_{n} & =\bar{\Lambda}\left(\bar{V}_{n-1}+\bar{V}_{n-1}\right) \\
& =2 \bar{\Lambda} \bar{V}_{n-1} \\
& =2^{n} \bar{\Lambda}^{n} \bar{V}_{0} .
\end{aligned}
$$

From this we see that if the largest absolute value of an eigenvalue of $2 \bar{\Lambda}$ is less than $1, \lim _{n \rightarrow \infty} \bar{V}_{n}$ will be the zero vector. This then implies that with probability one the number of infinite paths with sums bounded in the interval is zero. Now if we denote the entries of $\bar{\Lambda}$ by $\bar{\lambda}_{i j}$, we have that,

$$
\bar{\lambda}_{i j}=\left\{\begin{array}{cc}
p & i=j-1 \\
1-p & i=j+1 \\
0 & \text { otherwise }
\end{array}\right.
$$

It can be easily calculated that the largest eigenvalue of this matrix is $2 \sqrt{p(1-p)} \cos (\pi /(M+1))$, which gives the result.

For the proof of Theorem 1(b) we need the following combinatorial lemma, which we will prove at the end of this section. We denote by $N_{n}$ the number of oriented paths from $(0,0)$ to $(n, 2 n)$ with final partial sum zero. 
Lemma 3.1 We have, for $n$ even,

$$
\mathbb{P}_{p}\left(N_{n}>0\right) \geq \frac{\left(\begin{array}{c}
3 n \\
n
\end{array}\right)^{2}\left(\begin{array}{c}
3 n \\
3 n / 2
\end{array}\right)^{2}}{\sum_{k=1}^{3 n} T(n, k) \sum_{i=0}^{k}\left(\begin{array}{l}
k \\
i
\end{array}\right)\left(\begin{array}{c}
3 n-k \\
3 n / 2-i
\end{array}\right)^{2} p^{-i}(1-p)^{-k+i}},
$$

where

$$
T(n, k)=\sum_{a=0}^{3 n / 2} \sum_{m=0}^{k}\left(\begin{array}{c}
3 n-2 a \\
n-a
\end{array}\right) 2^{2 a} C(a, m)\left(\begin{array}{l}
k \\
m
\end{array}\right)\left(\begin{array}{c}
3 n-k-1 \\
3 n-2 a-(k-m)
\end{array}\right),
$$

and $C(a, m)$ is the coefficient of $t^{2 a}$ in the series expansion of $\left(1-\sqrt{1-t^{2}}\right)^{m}$.

With $n=14$ and $p \in(0.475,0.525)$ this gives us a lower bound of 0.934 .

Proof of Theorem 1(b) Lemma 3.1 shows that the probability of diagonally crossing a $n \times 2 n$ rectangle, with a final partial sum of zero is high. We then combine several of these events in a particular way, with a probability that is still high. Finally we place these rectangles onto a larger grid in such a way that we can show that this stochastically dominates supercritical ordinary site percolation on the square lattice. This then implies the existence, with positive probability, of a path with the required property.

We define, for even $n$, the following events $C_{1}, \ldots, C_{4}$.

$C_{1}=\{$ There exists an oriented path from $(0,0)$ to $(n, 2 n)$ with final partial sum 0$\}$,

$C_{2}=\{$ There exists an oriented path from $(0,0)$ to $(2 n, n)$ with final partial sum 0$\}$,

$C_{3}=\{$ There exists an oriented path from $(-2 n, n)$ to $(0,0)$ with final partial sum 0$\}$, 


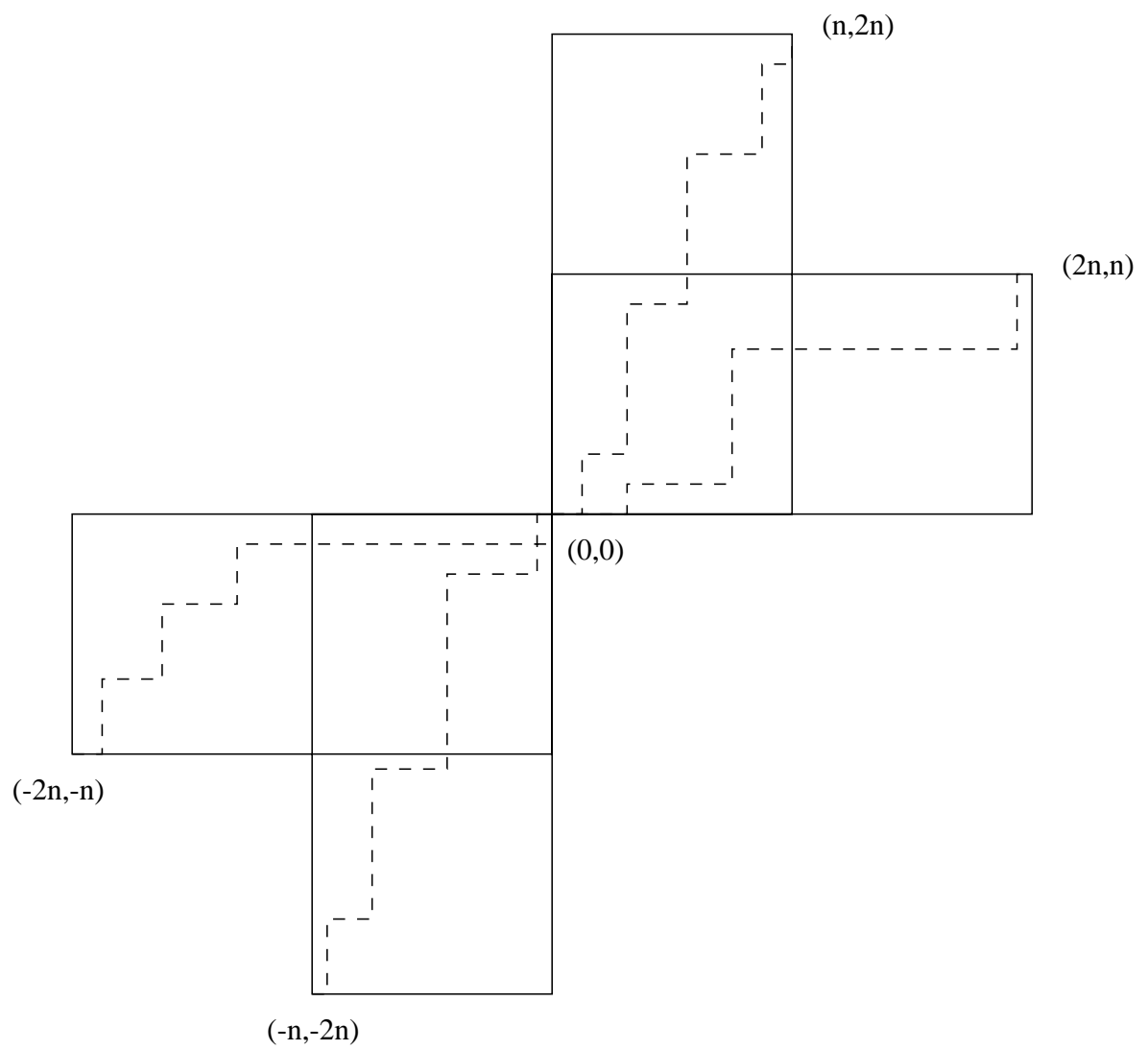

Figure 1: The event $C$; dashed lines indicate paths that cross the rectangles with final partial sums of zero

$C_{4}=\{$ There exists an oriented path from $(-n,-2 n)$ to $(0,0)$ with final partial sum 0$\}$.

The event $C=C_{1} \cap C_{2} \cap C_{3} \cap C_{4}$ then has probability at least $0.753>3 / 4$, for $n=14$ and $p \in(0.475,0.525)$ (using the fact that the events $C_{1}$ and $C_{2}$ are independent of $C_{3}$ and $C_{4}$ ). It is illustrated in Figure 1 . We denote by $w+C$ the event that $C$ occurs, but translated to $w$ from $(0,0)$.

We now move to the larger grid formed by the vertices $\{(2 a n, a n)+$ 


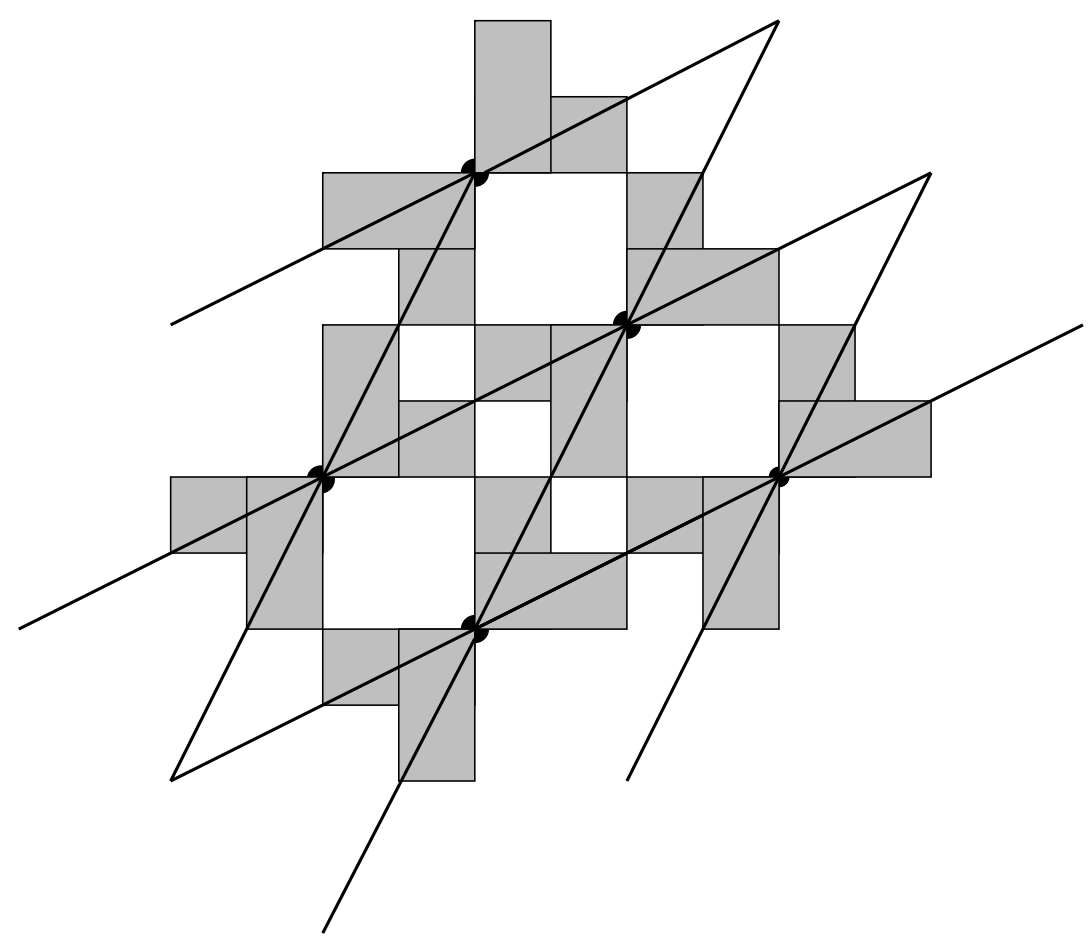

Figure 2: The larger grid; the grey areas are those we consider, heavy lines indicate the edges of the larger distorted lattice

$(b n, 2 b n): a, b \in \mathbb{Z}\}$ with edges from $(2 a n, a n)+(b n, 2 b n)$ to $(2(a+1) n,(a+1) n)+(b n, 2 b n)$ and $(2 a n, a n)+((b+1) n, 2(b+1) n)$. Note that this grid is a distorted version of the square lattice, and so has the same critical point for oriented independent percolation, which is known to be no more than $3 / 4$ (see [11]). We call a vertex, $(2 a n, a n)+(b n, 2 b n)$, on this grid 'open' if the event $(2 a n, a n)+(b n, 2 b n)+C$ occurs - see Figure 2.

A path of open vertices on the large grid then implies a path on the standard grid with partial sums that are zero every $3 n$ steps. This certainly happens with positive probability for $n=14$ and $p \in(0.475,0.525)$.

Finally we prove Lemma 3.1. 
Proof of Lemma 3.1 We shall estimate the probability that $N_{n}>0$ via a second moment method. The probability a particular oriented path from $(0,0)$ to $(n, 2 n)$ has a final partial sum of zero is $\left(\begin{array}{c}3 n \\ 3 n / 2\end{array}\right) p^{3 n / 2}(1-p)^{3 n / 2}$. There are $\left(\begin{array}{c}3 n \\ n\end{array}\right)$ of these paths, so that the expected value of $N_{n}$ is

$$
\left(\begin{array}{c}
3 n \\
n
\end{array}\right)\left(\begin{array}{c}
3 n \\
3 n / 2
\end{array}\right) p^{3 n / 2}(1-p)^{3 n / 2}
$$

Given two of these paths, if they meet at precisely $k$ vertices (excluding $(0,0))$, we claim that the probability that they both have partial sum zero at $(n, 2 n)$ is

$$
\sum_{i=0}^{k}\left(\begin{array}{l}
k \\
i
\end{array}\right)\left(\begin{array}{c}
3 n-k \\
3 n / 2-i
\end{array}\right)^{2} p^{3 n-i}(1-p)^{3 n-k+i}
$$

To see this, we condition upon the sum along the shared portion of the two paths. The probability this sum is equal to $j$ is $\left(\begin{array}{c}k \\ \frac{k+j}{2}\end{array}\right) p^{(j+k) / 2}(1-p)^{(k-j) / 2}$, when $\frac{k+j}{2}$ is an integer, and zero otherwise. The sums along the nonshared portions of the two paths are independent and each have probability $\left(\begin{array}{c}3 n-k \\ \frac{3 n-k-j}{2}\end{array}\right) p^{(3 n-k-j) / 2}(1-p)^{(3 n-k+j) / 2}$ of being equal to $-j$. Thus summing over the possible values of $j$ we find that the probability that both paths have partial sum zero at $(n, 2 n)$ is equal to

$$
\sum_{\substack{j=-k, j \text { with same parity as } k}}^{k}\left(\begin{array}{c}
k \\
\frac{k+j}{2}
\end{array}\right)\left(\begin{array}{c}
3 n-k \\
\frac{3 n-k-j}{2}
\end{array}\right)^{2} p^{\left(3 n-\frac{(k+j)}{2}\right)}(1-p)^{\left(3 n-\frac{(k-j)}{2}\right)} .
$$

If we now substitute $i$ for $\frac{j+k}{2}$, we find formula (2) above. 
Thus to calculate the expected value of $N_{n}^{2}$, we need to know only how many (ordered) pairs of oriented paths from $(0,0)$ to $(n, 2 n)$ there are that meet in precisely $k$ places (excluding $(0,0)$ ). If we denote this number by $T(n, k)$ then we have that,

$$
\mathbb{E}_{p}\left(N_{n}^{2}\right)=\sum_{k=1}^{3 n} T(n, k) \sum_{i=0}^{k}\left(\begin{array}{l}
k \\
i
\end{array}\right)\left(\begin{array}{c}
3 n-k \\
3 n / 2-i
\end{array}\right)^{2} p^{3 n-i}(1-p)^{3 n-k+i}
$$

We shall now first show that $T(n, k)$ is equal to the number of paths in $\mathbb{Z}^{2}$ with a certain property. Consider a pair $\left(\pi, \pi^{\prime}\right)$ of oriented paths in $\mathbb{Z}^{2}$ from $(0,0)$ to $(n, 2 n)$. We map (call this map $A$ ) this pair to an undirected path $\left(z_{0}, z_{1}, \ldots, z_{3 n}\right)$ in $\mathbb{Z}^{2}$ as follows. Start in the origin, that is, $z_{0}=$ $(0,0)$. The path is now constructed sequentially as follows. If $\pi$ and $\pi^{\prime}$ both make a step to the right, then $z_{1}=z_{0}+e_{1}$; if $\pi$ and $\pi^{\prime}$ both make a step upward, then $z_{1}=z_{0}-e_{1}$; if $\pi$ goes up, and $\pi^{\prime}$ goes to the right, then $z_{1}=z_{0}+e_{2}$; if $\pi$ goes to the right and $\pi^{\prime}$ goes upwards, then $z_{0}=z_{1}-e_{2}$. This procedure is repeated for each of the steps of $\pi$ and $\pi^{\prime}$. Here, $e_{1}$ and $e_{2}$ denote the unit vectors. For example, if $\pi=((0,0),(1,0),(1,1),(1,2))$ and $\pi^{\prime}=((0,0),(0,1),(0,2),(1,2))$ then we find the two dimensional path shown in Figure 3. Let us define a relevant path of length $3 n$ in $\mathbb{Z}^{2}$ to be a series of vertices $z_{0}, z_{1}, z_{2}, \ldots, z_{3 n}$ with $z_{0}=(0,0), z_{3 n}=(-n, 0)$ and $\left\|z_{i}-z_{i-1}\right\|=1, i=1,2, \ldots, 3 n$. Define a $k$-path of length $3 n$ as a relevant path of length $3 n$ in which precisely $k$ of the $z_{i}, i=1,2, \ldots, 3 n$, have second coordinate 0 . The map $A$ described above maps a pair of paths $\left(\pi, \pi^{\prime}\right)$ that meet in $k$ places to a $k$-path in a bijective fashion. We conclude that $T(n, k)$ is the number of $k$-paths of length $3 n$, and we shall now explain how we can find this number.

Given two one dimensional sequences, $y=\left(y_{0}, y_{1}, \ldots, y_{2 a}\right)$ (of length $2 a$ ) 


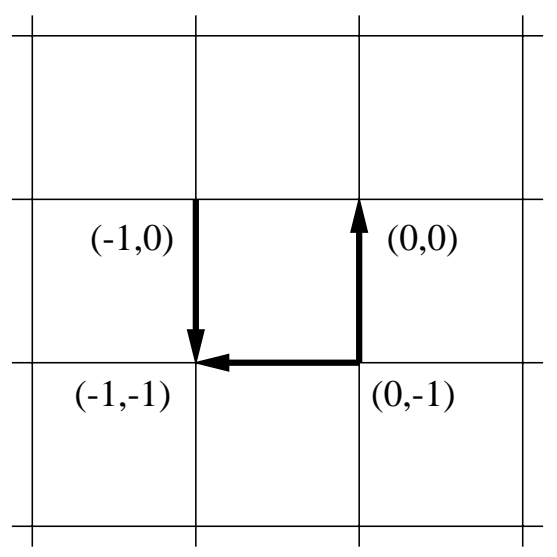

Figure 3: Path produced from $\pi=((0,0),(1,0),(1,1),(1,2))$ and $\pi^{\prime}=$ $((0,0),(0,1),(0,2),(1,2)$ by map $A$ or from $x=(0,-1), y=(0,-1,0)$, and $S=(v, h, v)$ by map $B$.

and $x=\left(x_{0}, x_{1}, \ldots, x_{3 n-2 a}\right)$ (of length $\left.3 n-2 a\right)$, both starting in 0 , and making steps of size \pm 1 , and a sequence $S=\left(s_{1}, s_{2}, \ldots, s_{3 n}\right), s_{i} \in\{v, h\}, i=$ $1,2, \ldots, 3 n$ containing $2 a v$ 's and $3 n-2 a h$ 's we can construct a path $z=\left(z_{0}, z_{1}, \ldots, z_{3 n}\right)$ of length $3 n$, in the following way. Start in the origin. We copy steps in $x$ to horizontal steps in $z$ and steps in $y$ to vertical steps in $z$, and the order in which this is done is governed by the order of the letters in $S$. For instance, if we take $x=(0,-1), y=(0,-1,0)$, and $S=(v, h, v)$ we again find the path in Figure 3 . We call this map $B$. It is easy to see that every relevant path can be produced from this procedure given the appropriate choice of $x, y$ and $S$, and that $B$ is injective.

Map $B$ produces a $k$-path of length $3 n$ from $y=\left(y_{0}, y_{1}, \ldots, y_{2 a}\right), x=$ $\left(x_{0}, x_{1}, \ldots, x_{3 n-2 a}\right)$ and $S$ if and only if all of the following hold, for some $m \leq k$ :

1. $y_{0}=y_{2 a}=0$ and $m$ of the steps of $y$ end at zero, that is, the set 
$\left\{0<i \leq 3 n-2 a ; y_{i}=0\right\}$ contains $m$ elements;

2. $x_{0}=0, x_{3 n-2 a}=-n$;

3. $k-m$ of the steps of $y$ either occur after a step of $x$ that ended at zero but before another step of $x$, or before any steps of $x$.

We shall now count how in how many ways this is possible. The probability generating function for the first return time for a one dimensional simple symmetric random walk is given by $\left(1-\sqrt{1-t^{2}}\right)$. Thus the probability that such a random walk returns to the origin for the $m$ th time at time $2 a$ is the coefficient of $t^{2 a}$ in $\left(1-\sqrt{1-t^{2}}\right)^{m}$. Denote this coefficient by $C(a, m)$. There are $2^{2 a}$ such simple symmetric random walk paths of length $2 a$, and they are all equally likely. Hence the number of paths $y$ above of length $2 a$ with precisely $m$ steps that end at zero is is $2^{2 a} C(a, m)$.

There are $\left(\begin{array}{c}3 n-2 a \\ n-a\end{array}\right)$ choices for $x$. Given $x$ and $y$, we need to find how many sequences $S$ of $h$ 's and $v$ 's give $k$-paths. Now $S$ must contain $2 a v ' s$, and we identify these, in order, with the $2 a$ steps of $y$ and label those that correspond to steps in $y$ that end at 0 with a star. We need to fit the remaining $3 n-2 a h$ 's into this sequence, such that $k-m$ of them appear either before any $v$, or after a starred $v$ but before any other $v$, and the other $3 n-2 a-(k-m)$ appear in other places. We claim that we can do this in $\left(\begin{array}{l}k \\ m\end{array}\right)\left(\begin{array}{c}3 n-k-1 \\ 3 n-2 a-(k-m)\end{array}\right)$ ways. This is the product of the number of ways in which $(k-m) h$ 's can be fitted into the $(m+1)$ places that are either before any $v$, or after a starred $v$ but before any other $v$ (denote this number by $W(k-m, m+1)$ ) and the number of ways in which the remaining $h$ 's can be fitted into the remaining $2 a-m$ places. To 
calculate $W(k-m, m+1)$ we work out the number of ways in which the $(k-m) h$ 's could be fitted into the $(m+1)$ places if the $h$ 's were labelled $h_{1}, h_{2}, \ldots, h_{k-m}$, and then divide by the number of labellings, $(k-m)$ ! We can put $h_{1}$ into $(m+1)$ places. There are then $(m+2)$ places into which we can put $h_{2}$, directly to either side of $h_{1}$ and into the places in which we did not put $h_{1}$. There are, similarly, $(m+3)$ places where we can put $h_{3}$, and as we continue in this manner, there will be $(m+i)$ places in which to put $h_{i}$. Hence $W(k-m, m+1)=\frac{(m+1)(m+2) \ldots(m+(k-m))}{(k-m) !}=\left(\begin{array}{l}k \\ m\end{array}\right)$. Counting the number of ways in which we can fit the rest of the $h$ 's into the other places works in exactly the same way. Thus if we sum over $m$ and $a$ we find the total number of $k$-paths,

$$
T(n, k)=\sum_{a=0}^{n} \sum_{m=0}^{k}\left(\begin{array}{c}
3 n-2 a \\
n-a
\end{array}\right) 2^{2 a} C(a, m)\left(\begin{array}{l}
k \\
m
\end{array}\right)\left(\begin{array}{c}
3 n-k-1 \\
3 n-2 a-(k-m)
\end{array}\right) .
$$

Finally, since $N_{n}$ is non-negative and integer-valued, the second moment bound

$$
\mathbb{P}\left(N_{n}>0\right) \geq \frac{\mathbb{E}_{p}\left(N_{n}\right)^{2}}{\mathbb{E}_{p}\left(N_{n}^{2}\right)}
$$

then gives the desired result.

\section{Proofs of Theorem 2 and Theorem 3}

The proofs of these results are not difficult and a re based on simple counting arguments. We will, however, need the following lemma. The proof of the lemma comes after the proof of the theorem.

Lemma 4.1 If, for a given value of $p$, there is an infinite self-avoiding path with bounded partial sums, with positive probability, then there is an 
infinite self-avoiding path whose partial sums returns to 0 infinitely often, with positive probability.

Proof of Theorem 2 We start by noting that if there exists a path with partial sums that return to 0 infinitely often, with positive probability, then the expected number of points that can be reached from 0 by a self-avoiding path with final partial sum 0 must be infinite.

However the expected number of points that can be reached from 0 by a self-avoiding path with final partial sum 0 is certainly no greater than the expected number of finite self-avoiding paths that have partial sum 0 on their last step. The number of self-avoiding paths of length $n$ is $\sigma_{G}(n)$. The probability that such a path has final partial sum 0 is $\left(\begin{array}{c}n \\ n / 2\end{array}\right) p^{n / 2}(1-p)^{n / 2}$ for $n$ even and 0 for $n$ odd. Thus this last expectation is,

$$
\begin{aligned}
& \sum_{n>0, n \text { even }} \sigma_{G}(n)\left(\begin{array}{c}
n \\
n / 2
\end{array}\right) p^{n / 2}(1-p)^{n / 2} \\
\leq & \sum_{\substack{n>0, n \text { even } \\
<\infty}} \sigma_{G}(n) 2^{n}(p(1-p))^{n / 2} \\
<\infty &
\end{aligned}
$$

for $p$ such that $2^{2} c_{G}^{2} p(1-p)<1$. Hence for these values of $p$ (which are those given in the theorem) there can be no path with partial sums that return to 0 infinitely often.

The part of the theorem referring to bounded partial sums then follows immediately from Lemma 4.1.

Proof of Lemma 4.1 We first show that

$$
\mathbb{P}_{p}\left(\exists \text { a path } \pi \text { with } S_{n}^{\pi}=0 \text { i.o. }\right)=0
$$


implies that for all $z \in \mathbb{Z}$,

$$
\mathbb{P}_{p}\left(\exists \text { a path } \pi \text { with } S_{n}^{\pi}=z \text { i.o. }\right)=0 .
$$

To see this, suppose that an integer, $z$ say, exists such that the event in (4) has positive probability. Then there must exist a non-random vertex, $x$ say, such that with positive probability, there exists a path which has partial sums that are $z$ infinitely often, passes through $x$, and has partial sum $z$ at $x$. Then we have a path from $x$ that has partial sums that are zero infinitely often. This contradicts (3).

Now, if we have an infinite self-avoiding path with partial sums that are bounded, then these partial sums must visit some value infinitely often. Thus, by the previous paragraph, if we have an infinite self-avoiding path with partial sums that remain bounded, with positive probability, we must have an infinite self-avoiding path with partial sums that are zero infinitely often, with positive probability.

Proof of Theorem 3 To prove the first part of this theorem we first fix $p \in(0,1)$. We can find a box size, $m$, such that with probability higher than $3 / 4$ the box

\[ (m)=\{(x, y): x, y \in\{0,1, \ldots, m-1\}\} \]
contains the configuration $\left\{\begin{array}{ll}-1 & 1 \\ -1 & 1\end{array}\right\}$. We call this a balancing configuration. Now divide the square lattice into boxes such that $(x, y)$ is in the box indexed by $(a, b), a, b \in \mathbb{Z}$ if $x \in\{m a, m a+1, \ldots, m(a+1)-1\}$ and $y \in\{m b, m b+1, \ldots, m(b+1)-1\}$. Call the box indexed by $(a, b)$ 'open' if it contains the above balancing configuration, and 'closed' otherwise. These events are independent and have identical probability for all boxes. The fact that we have site percolation on the oriented lattice when boxes are open 
with probability greater than $3 / 4$ now implies that with positive probability we have an infinite sequence of adjacent (that is, sharing one boundary edge) open boxes (see [11]). We now show that this event implies the existence of a just-visiting path that has bounded partial sums returning to 0 infinitely often.

Our path takes the shortest route from balancing configuration in one open box to balancing configuration in the next, in the infinite sequence of adjacent open boxes. When at a balancing configuration the path moves about on the -1 s or 1 s until its partial sum becomes zero. When this happens it goes to the next open box in the path. The partial sums never leave the interval $[-3 m, 3 m]$, so we have our path.

For the second part of the theorem we use a renormalisation argument, and then count paths. We will show that for $p \in(\sqrt[(2 N+1)]{2} \sqrt{6 / 7}, 1]$ we can have no paths with bound $N$, from which the result follows.

Fix $N$. We will use the enhanced square lattice, which is obtained from the ordinary square lattice by adding edges between all pairs of vertices at Euclidean distance $\sqrt{2}$. We divide the square lattice into disjoint boxes, $\{B(N+1)+((N+1) a,(N+1) b): a, b \in \mathbb{Z}\}$. We call a box 'open' if it contains a vertex with a -1 assigned to it, and 'closed' otherwise. Now if there exists a circuit of adjacent closed boxes around the origin then there can be no path from the origin with partial sums bounded in some interval of length $N$. There is such a circuit of adjacent closed boxes surrounding the origin if and only if there is no infinite self-avoiding path of open boxes in the enhanced square lattice. Note that the number of self-avoiding paths of length $n$ on the enhanced square lattice is less than $8 \times 7^{n}$. The probability that a box is closed is $p^{(2 N+1)^{2}}$. Hence the probability to have a path of length $n$ of 
open boxes in the enhanced lattice is at most $8 \times 7^{n}\left(1-p^{(2 N+1)^{2}}\right)^{n}$. This tends to zero, as $n \rightarrow \infty$, in the range indicated in the theorem.

\section{$5 \quad$ Proofs of Theorem 4 and Corollary 5}

Before we start with the involved proof of the theorem, it is worth to explain why the result is reasonable. When $p$ is close to 1 , there will be mostly +1 s around with isolated -1 s every now and then. Therefore, partial sums will typically increase. We can essentially only decrease partial sums along a just-visiting path, when we see two neighbouring vertices both with label -1 . Therefore, these double -1 s play an important role, and the only way to keep the partial sums bounded is to jump around from one such double -1 to the other. For $p$ close to 1 , the spatial distribution of these double -1 s will be close to a Poisson process. Depending on the density of these double -1 s it will or will not be possible for a sequence of partial sums to stay within a given bound. This is where continuum percolation comes in. If the double $-1 \mathrm{~s}$ are too far apart from each other, that is, if a certain percolation process does not percolate, then the bound will be large. If the percolation process does percolate, then we can use an infinite path in the percolation process to find a path with partial sums that remain bounded.

A significant amount of work is necessary to turn this idea into a proof. The connection with continuum percolation is made in Proposition 5.1, Lemma 5.2 and Lemma 5.6. In Lemma 5.3 and Lemma 5.7 this is translated into statements concerning the existence of paths with bounded partial sums for particular values of the parameter $p$. In the final proof of the theorem we then show how we can obtain the general result.

Fix $\lambda>0$. On the grid $(\mathbb{Z} / N)^{2}$ assign the value 1 to each vertex with prob- 
ability $p_{N}$, and the value -1 otherwise, independently of all other vertices, and where $p_{N}$ is defined as to satisfy

$$
2 N^{2}\left(1-p_{N}\right)^{2}=\lambda
$$

Next we put a point at the centre of every edge of the grid which has -1 s at both ends, and the resulting points form a point process which we call $\Pi_{N}$. We shall also need the point process $\Pi_{N}^{+}$defined as follows. Consider an edge which contains a point of $\Pi_{N}$. This point is in $\Pi_{N}^{+}$if and only if the parallel edge immediately to the right or above it has +1 s at its endpoints. We call such a configuration of two adjacent -1 s with two adjacent +1 s to the right or above it a balancing configuration. $\Pi_{N}^{+}$contains no other points, hence $\Pi_{N}^{+}$is a subset of $\Pi_{N}$. We write $\Pi_{N}(S)$ to denote the number of points of $\Pi_{N}$ in $S$. The number of points of $\Pi_{N}$ in $S$ that are due to edges that are completely contained in $S$ is denoted by $\Pi_{N}\left(S^{-}\right)$. Similar definitions apply to $\Pi_{N}^{+}$.

Proposition 5.1 The point processes $\Pi_{N}$ converge weakly to a homogeneous Poisson process with rate $\lambda$ when $N \rightarrow \infty$.

To prove this more or less obvious result, it suffices to prove convergence of the avoidance function, that is, the probability that a finite union of rectangles contains no points, see [6], Proposition 9.1.IX. This can be done directly from the definition of the $p_{N}$ s. However, in the proof of the forthcoming Lemma 5.6 we shall need a estimate about weak convergence that is uniform for $\lambda$ in a given interval. For that estimate we shall use an explicit Stein-Chen upper bound on the total variation distance between two distributions. As a warm-up we shall also use this Stein-Chen approach in the following proof of Proposition 5.1. 
Proof of Proposition 5.1 Consider a union of rectangles $R$ which, without loss of generality, we will assume has total area 1 . We say that en edge $e$ satisfies $e \in R$ if the centre of $e$ is contained in $R$. We write $G_{e}$ for the event that $e$ has -1 s on both endpoints. Now $\Pi_{N}(R)$ can be written as

$$
\Pi_{N}(R)=\sum_{e \in R} 1_{G_{e}}
$$

where $1_{G_{e}}$ denotes the indicator function of $G_{e}$. This indicator function is increasing in the number of $-1 \mathrm{~s}$, and has expectation $\left(1-p_{N}\right)^{2}$. We can therefore apply Corollary 2.E. 1 in [4] and conclude that the total variation distance between a Poisson distribution with parameter $\mathbb{E}_{p}\left(\Pi_{N}(R)\right)$ and $\Pi_{N}(R)$ is bounded above by

$$
\frac{1-e^{-\mathbb{E}_{p}\left(\Pi_{N}(R)\right)}}{\mathbb{E}_{p}\left(\Pi_{N}(R)\right)}\left(\operatorname{Var}\left(\Pi_{N}(R)\right)-\mathbb{E}_{p}\left(\Pi_{N}(R)\right)+2 \sum_{e \in R}\left(1-p_{N}\right)^{4}\right) .
$$

The asymptotic variance of $\Pi_{N}(R)$ is easily computed: $\Pi_{N}(R)$ is a sum of $(2+o(1)) N^{2}$ indicator random variables associated with the edges of the grid. When these edges are not adjacent, the corresponding indicator random variables are independent. Each edge (apart from those at the boundary which have fewer) has 6 adjacent edges, and the probability that two adjacent edges both have a point from $\Pi_{N}$ is equal to $\left(1-p_{N}\right)^{3}$. These observations lead to

$$
\begin{aligned}
\mathbb{E}_{p_{N}}\left(\Pi_{N}(R)^{2}\right)= & (2+o(1)) N^{2}\left(1-p_{N}\right) 2+6(2+o(1)) N^{2}\left(1-p_{N}\right)^{3}+ \\
& +(2+o(1))^{2} N^{4}\left(1-p_{N}\right)^{4} \\
\rightarrow & \lambda+\lambda^{2}
\end{aligned}
$$

when $N \rightarrow \infty$, because $\lambda=2 N^{2}\left(1-p_{N}\right)^{2}$. Clearly, $\lim _{N \rightarrow \infty} \mathbb{E}_{p}\left(\Pi_{N}(R)\right)=\lambda$. 
Hence $\operatorname{Var}\left(\Pi_{N}(R)\right)$ converges to $\lambda$ and since

$$
\sum_{e \in R}\left(1-p_{N}\right)^{4}=(2+o(1)) N^{2}\left(1-p_{N}\right)^{4} \rightarrow 0,
$$

for $N \rightarrow \infty$, the bound (6) above implies that the total variation distance between the distribution of $\Pi_{N}(R)$ and a Poisson distribution with parameter $\mathbb{E}_{p}\left(\Pi_{N}(R)\right)$ converges to 0 . A Poisson distribution with parameter $\mathbb{E}_{p}\left(\Pi_{N}(R)\right)$ certainly converges in distribution to a Poisson distribution with parameter $\lambda$, and as convergence in total variation implies convergence in distribution, we have that $\Pi_{N}(R)$ converges in distribution to a Poisson distribution with parameter $\lambda$.

Next we centre a diamond with radius $1 / 2$ around each point of $\Pi_{N}$. The result we shall call the discrete diamond process, to distinguish it from the continuous diamond process which is similar, but with diamonds centred around points of a Poisson process. The process obtained by centring diamonds around points of $\Pi_{N}^{+}$is called the reduced discrete diamond process. Recall the definition of $\lambda_{c}$ as the critical density associated with the continuous diamond process. The reason for the slightly larger radius in the next lemma will become apparent soon.

\subsection{Proof of Theorem 4(a)}

We now first prove the subcritical part of the theorem.

Lemma 5.2 Let $\lambda<\lambda_{c}$. There exists $\epsilon>0$ such that for all $\delta>0$ we can find $L$ sufficiently large such that for all large $N$, the probability (under $\mathbb{P}_{p_{N}}$ ) that the discrete diamond process with radii $\frac{1}{2}(1+\epsilon)$ connects $[0,3 L] \times 0$ to $[0,3 L] \times L$ within $[0,3 L] \times[0, L]$ is at most $\delta$. 
Proof Let $\lambda<\lambda_{c}$. Then using scaling relation (1) we can find $\epsilon>0$ such that $\lambda<\lambda_{c}\left(\frac{1}{2}(1+\epsilon)\right)$. In words, after increasing the radii of the diamonds by a factor $1+\epsilon$, the continuous diamond process remains subcritical. Now let $\delta>0$. Since the continuous diamond process (with increased radii) is subcritical, we have from Theorem 3.5 in [13] (stated for balls but the proof also works for diamonds) that the probability that this continuous diamond process connects $[0,3 L] \times 0$ to $[0,3 L] \times L$ is at most $\delta / 2$, for $L$ sufficiently large. We fix such $L$. Choose $\epsilon^{\prime}$ so small that if we partition $[0,3 L] \times[0, L]$ into squares with side length $\epsilon^{\prime}$, with probability at least $1-\delta / 2$ we have that

(i) a Poisson process with rate $\lambda$ has at most one point in each of the squares,

(ii) the connectivity structure of the diamonds (of radius $\frac{1}{2}(1+\epsilon)$ ) around these points does not change when we move points of the Poisson process around in the squares with side length $\epsilon^{\prime}$ in which they are contained.

We denote this set of squares with side length $\epsilon^{\prime}$ by $\mathcal{S}$. Since $\Pi_{N}$ converges weakly to a Poisson process with rate $\lambda$ (Proposition 5.1), the $\mathbb{P}_{p_{N^{-}}}$ probability that a particular subset of $\mathcal{S}$ contains a point of $\Pi_{N}$, converges to the corresponding probability in a Poisson process with rate $\lambda$. Property (ii) above than guarantees that if the continuous diamond process does not connect opposite sides, neither does the discrete one.

We next turn our previous lemma into a statement about paths with bounded partial sums. 
Lemma 5.3 For $\lambda<\lambda_{c}$, there exists $N^{\prime \prime}$ such that for all $N>N^{\prime \prime}$ there are $\mathbb{P}_{p_{N}}$-almost surely no just-visiting paths with partial sums bounded in $[0, N]$.

For the proof of Lemma 5.3, we need some more propositions. The $\epsilon$ in the statements that follow is the $\epsilon$ dictated by $\lambda$ in the statement of Lemma 5.2. If the discrete diamond process with increased radii does not connect the top and bottom sides of $[0,3 L] \times[0, L]$, we say that there is a gap from $0 \times[0, L]$ to $3 L \times[0, L]$. Other gaps are defined analogously. Lemma 5.2 says that the probability of a gap can be made as high as desired by taking $L$ large (and large $N$ of course).

Proposition 5.4 For $\delta>0$ and $\lambda<\lambda_{c}$ there exists $L$, such that for large $N$, with $\mathbb{P}_{p_{N}}$-probability at least $1-\delta$ we can construct a (random) pair of curves, $C_{1}$ and $C_{2}$ contained in $[0,3 L] \times[0, L]$ such that

1. the curves are self-avoiding and made up of finitely many straight line segments,

2. the curves start in $0 \times[0, L]$ and end in $3 L \times[0, L]$,

3. $C_{2}$ is the locus of points at distance $(1+\epsilon)$ from $C_{1}$, above $C_{1}$,

4. the conditional (that is, given $C_{1}$ and $C_{2}$ ) joint distribution of the configuration between $C_{1}$ and $C_{2}$ is i.i.d. with the original marginals, conditioned on the event that there are no adjacent -1 s.

Proof According to the proof of Lemma 5.2 there exists $L$ such that the probability of having an overlapping series of diamonds connecting top and 
bottom of the box $[0,3 L] \times[0, L]$ is smaller than $\delta / 6$, and we fix a large such $L$.

According to Lemma 5.2, we can now choose $N^{\prime}$ such that if we put a diamond of radius $\frac{1}{2}(1+\epsilon)$ at every pair of adjacent $-1 \mathrm{~s}$, the probability that these connect top and bottom of $[0,3 L] \times[0, L]$ is smaller than $\delta / 2$. Thus $C_{1}$ and $C_{2}$ with the first three properties above must exist with probability at least $1-\delta / 3$.

We now show that we can construct such a gap, without finding any more information about those $1 \mathrm{~s}$ and $-1 \mathrm{~s}$ in the gap other than that there are no adjacent pairs of $-1 \mathrm{~s}$, and possibly that some of the vertices on the edge of the gap have value 1 . We will need to work on the extended rectangle $[-(1+\epsilon), 3 L+(1+\epsilon)] \times[0, L]$, to take into account the dependencies between different areas. The probability that there is a gap crossing this extended rectangle is greater than $1-\delta$ for large $L$. This can easily be seen by combining horizontal gaps in $[-(1+\epsilon), 3 L-(1+\epsilon)] \times[0, L]$ and $[+(1+$ $\epsilon), 3 L+(1+\epsilon)] \times[0, L]$ with a vertical gap crossing $[0, L]^{2}$. Each of these gaps exists with probability at least $1-\delta / 3$, which means that the probability that they all exist must be at least $1-\delta$. Any gap crossing this rectangle clearly contains a gap crossing $[0,3 L] \times[0, L]$.

Order the edges in the extended rectangle $[-(1+\epsilon), 3 L+(1+\epsilon)] \times[0, L]$, in some deterministic way and call this set $E_{L}$. Until the end of this proof all edges mentioned are assumed to be in $E_{L}$. If we have an edge $e_{a}$ with $-1 \mathrm{~s}$ at both ends, we say that another edge $e_{b}$ is in the cluster of $e_{a}$ if there is a sequence of adjacent edges $\left(e_{a}=\epsilon_{0}, e_{1}, \ldots, \epsilon_{k}=e_{b}\right)$ all having $-1 \mathrm{~s}$ at both ends.

Set $E_{B}^{0}$ to be those (random) edges on the horizontal line closest to $[-(1+\epsilon), 3 L+(1+\epsilon)] \times 0$ with -1 s at both ends, along with all the edges 
in the clusters containing these edges. Let $E_{G}^{0}$ be the set of those edges in $E_{L}$ that lie on the same horizontal line and do not have -1 s at both ends, along with any edges adjacent to those in $E_{B}^{0}$ yet not in it.

In an inductive fashion, to find $E_{G}^{i+1}$ and $E_{B}^{i+1}$ from $E_{G}^{i}$ and $E_{B}^{i}$ do the following. Take the first edge in $E_{G}^{i}$ in the ordering, and check all edges in $E_{L} \cap\left(E_{G}^{i} \cup E_{B}^{i}\right)^{c}$ completely within distance $(1+\epsilon)$ of this edge to see if they have $-1 \mathrm{~s}$ at both ends. If such an edge exists, set $E_{B}^{i+1}$ to be $E_{B}^{i}$ along with all such edges and any edges in their clusters around them. In addition, set $E_{G}^{i+1}$ to be $E_{G}^{i}$ along with any edges sharing a vertex with an edge in $E_{B}^{i+1}$, yet not in it. In the case that the first edge in $E_{G}^{i}$ has no edges in $E_{L} \cap\left(E_{G}^{i} \cup E_{B}^{i}\right)^{c}$ within distance $(1+\epsilon)$ with a -1 at both ends, move through the ordering of edges until either an edge is found that does have this property or there are no more edges in $E_{G}^{i}$. In the first case proceed as we would have done for the first edge, except with this edge, and in the latter case set $E_{G}^{i+1}$ and $E_{B}^{i+1}$ to be $E_{G}^{i}$ and $E_{B}^{i}$.

There must be a value of $i$ for which $E_{G}^{i}$ and $E_{G}^{i+1}$ are the same, as are $E_{B}^{i}$ and $E_{B}^{i+1}$, if only because there are only finitely many vertices in the box. At this point either there is an edge in $E_{G}^{i}$ which is closer than $(1+\epsilon)$ to the top of the box, or not. If this is so there can be no gap of size $(1+\epsilon)$ in the box by the manner of our construction, and if this is not so we must have found a gap (between the locus of points at least $(1+\epsilon)$ away from all the edges $E_{G}$ and the points $(1+\epsilon)$ from this locus back towards the $\left.E_{G} s\right)$. What do we know about the points in our gap? The ends of the edges in $E_{G}$ closest to the edges in $E_{B}$ must have value 1, but apart from this, our construction method has told us exactly that there are no pairs of $-1 \mathrm{~s}$ in the gap. 
Proposition 5.5 Given $\delta>0$ and $\lambda<\lambda_{c}$ there exists $N^{\prime \prime}$ and $L$ such that, for all $N>N^{\prime \prime}$, the probability (under $\mathbb{P}_{p_{N}}$ ) that there exists a path with sums bounded in $[0, N]$ from $[0,3 L] \times 0$ to $[0,3 L] \times L$ in $[0,3 L] \times[0, L]$ is less than $\delta$.

Proof Take $N, L$ so that the probability that a gap exists is at least $1-\delta / 2$. Then if there is a gap from $0 \times[0, L]$ to $3 L \times[0, L]$, then there exist curves $C_{1}$ and $C_{2}$ as in Proposition 4. In order to have a path with sums bounded in $[0, N]$ from $[0,3 L] \times 0$ to $[0,3 L] \times L$ in $[0,3 L] \times[0, L]$, there must exist at least one path from $C_{1}$ to $C_{2}$ with partial sum bounded above by $N$. Since there are no adjacent $-1 \mathrm{~s}$ between $C_{1}$ and $C_{2}$, if such a path exists, then also a selfavoiding path from $C_{1}$ to $C_{2}$ with this property exists: indeed, in the absence of adjacent -1 s, the final partial sum cannot be made less by adding loops. Denote the number of self-avoiding paths between $C_{1}$ and $C_{2}$ with partial sums bounded above by $N$ by $U_{N}$. We call an event increasing if its indicator function is non-decreasing in the natural partial ordering of configurations of $\pm 1 \mathrm{~s}$, and we call it decreasing if its complement is increasing. We are interested in the decreasing event $\left\{U_{N}>0\right\}$ conditioned on the increasing event of having no adjacent -1 s between the curves. The standard FKG inequality (see for instance [9]) then implies that the conditional probability of the event $\left\{U_{N}>0\right\}$ is at most the unconditional probability of the same event, that is, its probability under $\mathbb{P}_{p_{N}}$. Note that such a path, of length $M$ say, must contain at least $\frac{M-N}{2}-1 \mathrm{~s}$, if its partial sums are to be bounded above by $N$. Hence the conditional probability that $U_{N}>0$ is bounded above by

$$
\sum_{M \geq(1+\epsilon) N} 3 L^{2}(N+1)^{2} 4 \cdot 3^{M-1} \sum_{j=(M-N) / 2}^{M}\left(\begin{array}{c}
M \\
j
\end{array}\right) p_{N}^{M-j}\left(1-p_{N}\right)^{j}
$$




$$
\begin{aligned}
& \leq \sum_{M \geq(1+\epsilon) N} 3 L^{2}(N+1)^{2} 4 \cdot 3^{M-1}\left(1-p_{N}\right)^{\frac{M-N}{2}} 2^{M} \\
& =\frac{4 L^{2}(N+1)^{2}\left(1-p_{N}\right)^{\epsilon N / 2} 6^{(1+\epsilon) N}}{1-6\left(1-p_{N}\right)^{1 / 2}}
\end{aligned}
$$

This converges to zero as $N \rightarrow \infty$. Thus we can find $N^{\prime}$ such that for $N>N^{\prime}$ this is less than $\delta / 2$.

Proof of Lemma 5.3 First choose $\delta$ and then define the event the $(0,0)$ is closed as the occurrence of all of the following events,

- there is a gap from $0 \times[0, L]$ to $3 L \times[0, L]$ which is not crossed by a path with partial sums bounded in $[0, N]$,

- there is a gap from $[0, L] \times 0$ to $[0, L] \times 3 L$ which is not crossed by a path with partial sums bounded in $[0, N]$,

- there is a gap from $0 \times[2 L, 3 L]$ to $3 L \times[2 L, 3 L]$ which is not crossed by a path with partial sums bounded in $[0, N]$,

- there is a gap from $[2 L, 3 L] \times 0$ to $[2 L, 3 L] \times 3 L$ which is not crossed by a path with partial sums bounded in $[0, N]$.

Note that by the previous proposition there exists $N^{\prime}, L$, so that this occurs with probability at least $1-4 \delta$, for $N>N^{\prime}$. We now ask whether this event occurs at other points, in particular at $\{(2 a L, 2 b L): a, b \in \mathbb{Z}\}$, in which case we say $(a, b)$ is closed. These events are not independent, but the events ' $(a, b)$ is closed' and ' $(c, d)$ is closed' are independent if ( $2 a L-$ $2 c L)^{2}+(2 b L-2 d L)^{2}>8 L^{2}$. This means we can compare this process to a 1-dependent ordinary site percolation model on the enhanced square lattice. A standard counting argument (see for instance [10]) shows that for $\delta$ sufficiently small, there can be no infinite self-avoiding path of non-closed points. 
Any path with partial sums that are bounded in $[0, N]$ cannot pass through gaps without using a section of path that is itself bounded in $[0, N]$. Thus it must be contained in the non-closed areas, which are, by the above argument, almost surely finite. Therefore it cannot be infinite itself, almost surely.

Proof of Theorem 4(a) If $\limsup _{N \rightarrow \infty} 2 N^{2}\left(1-q_{N}\right)^{2}<\lambda_{c}$, then we can find $N_{0}$, and $\lambda$ such that for all $N>N_{0}$,

$$
2 N^{2}\left(1-q_{N}\right)^{2}<\lambda<\lambda_{c}
$$

If we use this $\lambda$ in Lemma 5.3, we see that there can be no paths with partial sums bounded in $[0, N]$ for $N$ large enough, almost surely, for the sequence of $p_{N} \mathrm{~s}$, defined by this $\lambda$. However for $N>N_{0}, p_{N}<q_{N}$, which means that we can couple the two discrete processes with parameters $p_{N}$ and $q_{N}$ in the natural way, that is, the set of vertices with value -1 under $q_{N}$ is a subset of the corresponding set under $p_{N}$. Now it is a matter of carefully inspecting the proof of Lemma 5.3. We first showed that the limit point process $\Pi_{N}$ is a Poisson process. The only place where this fact was used later was to assert that appropriate gaps exist in the subcritical Boolean model. The coupling just mentioned implies that point processes $\Pi_{N}^{\prime}$ associated with the $q_{N} s$ will be stochastically smaller than $\Pi_{N}$. (Note that we do not have information about a possible weak limit of the $\Pi_{N}^{\prime} s$.) This means that gaps have an even higher probability to occur with the $q_{N} s$ than with the $p_{N} s$. The estimate of the probability to bridge such a gap with bounded partial sums is monotone in $p_{N}$ for $N$ sufficiently large. 


\subsection{Proof of Theorem 4(b)}

The proof of the supercritical part of the theorem must be different as we will need to consider balancing configurations (recall the definition above) the occurrence of which, as they contain both $1 \mathrm{~s}$ and $-1 \mathrm{~s}$, is neither increasing nor decreasing in $p$. We begin by considering percolation of diamonds of the reduced discrete diamond process. In the next lemma, the uniformity of $N_{1}$ in $\lambda$ will be important later on.

Lemma 5.6 Given $\lambda_{c}<\lambda_{\text {down }}<\lambda_{\text {up }}<\infty$. There exists $N_{1}$ such that for all $N>N_{1}$ and $\lambda$ such that $\lambda_{\text {down }}<\lambda<\lambda_{\text {up }}$ the reduced discrete diamond process percolates under $\mathbb{P}_{p_{N}}$.

Proof of Lemma 5.6 We can choose $\epsilon>0$ such that $\lambda_{\text {down }}(1-\epsilon)^{3}>\lambda_{c}$. Rearranging and using scaling relation (1) above we find that, $\lambda_{\text {down }}(1-\epsilon)>$ $\lambda_{c}\left(\frac{1}{2}(1-\epsilon)\right)$. In words, after decreasing both the rate of the Poisson process and the radii of the diamonds by a factor $1-\epsilon$, the continuous diamond process remains supercritical. It is clear that this is also true (with the same $\epsilon$ ) for any $\lambda>\lambda_{\text {down }}$.

Now consider the grid $\left(\frac{1}{4} \epsilon \mathbb{Z}\right)^{2}$. A square of this grid is called a grid square. Any diamond of radius $\frac{1}{2}(1-\epsilon)$ centred in a grid square is then contained in any diamond of radius $\frac{1}{2}$ centred in that same grid square. For a grid square $S$, we denote by $F_{S}$ the event that $\Pi_{N}^{+}\left(S^{-}\right) \geq 1$. We first claim that for $N \rightarrow \infty$

$$
\mathbb{P}_{p_{N}}\left(F_{S}\right) \rightarrow 1-e^{-\lambda(\epsilon / 4)^{2}},
$$

uniformly in $\lambda_{\text {down }}<\lambda<\lambda_{\text {up }}$. For this we use the Stein-Chen Poisson approximation anticipated in the proof of Proposition 5.1 to estimate the 
total variation distance between the distributions of the number of points in $S$ from $\Pi_{N}$ and that from a Poisson distribution of rate corresponding to the expected number of such points, $\mathbb{E}_{p}\left(\Pi_{N}(S)\right)$. As in $(6)$, we have that this total variation distance is at most

$$
\frac{1-e^{-\mathbb{E}_{p}\left(\Pi_{N}(S)\right)}}{\mathbb{E}_{p}\left(\Pi_{N}(S)\right)}\left(\operatorname{Var}\left(\Pi_{N}(S)\right)-\mathbb{E}_{p}\left(\Pi_{N}(S)\right)+2 \sum_{\text {edges in } S}\left(1-p_{N}\right)^{4}\right) .
$$

A similar albeit somewhat more complicated computation as in the proof of Proposition 5.1 now yields that this is bounded above by

$$
\begin{aligned}
& \frac{1-e^{-2(N+1)^{2}\left(\frac{\epsilon}{4}\right)^{2}\left(1-p_{N}\right)^{2}}}{2 N^{2}\left(\frac{\epsilon}{4}\right)^{2}\left(1-p_{N}\right)^{2}} \times \\
& \quad\left(6 \cdot 2\left(\frac{\epsilon}{4}\right)^{2}(N+1)^{2}\left(1-p_{N}\right)^{3}+2 \cdot 2\left(\frac{\epsilon}{4}\right)^{2}(N+1)^{2}\left(1-p_{N}\right)^{4}\right)
\end{aligned}
$$

Keeping in mind relation (5), this then gives the bound

$$
\frac{1-e^{-\left(\frac{N+1}{N}\right)^{2}\left(\frac{\epsilon}{4}\right)^{2} \lambda}}{\left(\frac{\epsilon}{4}\right)^{\lambda}}\left(6 \cdot \frac{(N+1)^{2}}{N^{3}}\left(\frac{\epsilon}{4}\right)^{2} \frac{\lambda^{3 / 2}}{\sqrt{2}}+2 \cdot \frac{(N+1)^{2}}{N^{4}}\left(\frac{\epsilon}{4}\right)^{2} \lambda^{2}\right)
$$

This tends to zero uniformly in $\lambda_{\text {down }}<\lambda<\lambda_{\text {up }}$ when $N \rightarrow \infty$. The fact that

$$
\left(\frac{\epsilon}{4}\right)^{2} \lambda \leq \mathbb{E}_{p}\left(\Pi_{N}(S)\right) \leq\left(\frac{N+1}{N}\right)^{2}\left(\frac{\epsilon}{4}\right)^{2} \lambda
$$

implies that the null probability of Poisson distribution of rate $\mathbb{E}_{p}\left(\Pi_{N}(S)\right)$ converges to the null probability of a Poisson distribution of rate $\left(\frac{\epsilon}{4}\right)^{2} \lambda$. Claim (7) follows immediately.

Next we want to show that

$$
\mathbb{P}_{p_{N}}\left(\left\{\Pi_{N}^{+}\left(S^{-}\right)=0\right\} \cap\left\{\Pi_{N}\left(S^{-}\right)>0\right\}\right) \rightarrow 0,
$$


uniformly in $\lambda_{\text {down }}<\lambda<\lambda_{\text {up }}$, as $N \rightarrow \infty$. The event in (8) can only occur if we see one of the configurations

$$
\left\{\begin{array}{rr}
-1 \\
-1 & -1
\end{array}\right\},\left\{\begin{array}{ll}
-1 & \\
-1 & -1
\end{array}\right\},\left\{\begin{array}{ll}
-1 & -1 \\
-1 &
\end{array}\right\}
$$

somewhere in the grid square. The probability of this is at most $3(\epsilon N / 4+$ $1)^{2}\left(1-p_{N}\right)^{3}=(\epsilon N / 4+1)^{2}\left(\frac{\lambda}{2}\right)^{3 / 2} \frac{1}{N^{3}}$. This goes to zero uniformly for all $\lambda_{\text {down }}<\lambda<\lambda_{\text {up }}$, when $N \rightarrow \infty$.

According to (7) and (8), we find that uniformly in $\lambda_{\text {down }}<\lambda<\lambda_{\text {up }}$, for all $N$ large enough,

$$
\mathbb{P}_{p_{N}}\left(F_{S}\right)>1-e^{-\lambda(1-\epsilon)(\epsilon / 4)^{2}},
$$

which is the probability that there is a point in the grid square due a Poisson point from a Poisson process of rate $\lambda(1-\epsilon)$. Hence we can couple the continuous diamond process based on this Poisson process and the reduced discrete diamond process in such a way that whenever a grid square contains at least one Poisson point, it also contains at least one point of $\Pi_{N}^{+}$. The grid size was chosen in such a way that the union of the diamonds (of radius $\left.\frac{1}{2}(1-\epsilon)\right)$ of the Poisson points, are contained in the union of the diamonds (with radius $\frac{1}{2}$ ) corresponding to $\Pi_{N}^{+}$. The former process is supercritical by the choice of $\epsilon$, and therefore also the latter process is supercritical, which is what we wanted to prove.

We next turn this lemma into a statement of paths with bounded partial sums.

Lemma 5.7 Given $\lambda_{c}<\lambda_{\text {down }}<\lambda_{\text {up }}<\infty$ there exists $N_{1}$ such that for all $N>N_{1}$ and $\lambda$ such that $\lambda_{\text {down }}<\lambda<\lambda_{\text {up }}$ there is a just-visiting path with partial sums bounded in $[0, N+1]$ with positive $\mathbb{P}_{p_{N}}$ probability. 
Proof of Lemma 5.7 Fix $\lambda$ such that $\lambda_{\text {down }}<\lambda<\lambda_{\text {up }}$. Recall that $2 N^{2}\left(1-p_{N}\right)^{2}=\lambda$. Then, By Lemma 5.6, the origin is contained in an unbounded connected components of diamonds from the reduced discrete diamond process with positive probability. If this is the case, we have a self-avoiding path $\pi=\left(\pi_{0}=0, \pi_{1}, \ldots\right)$ on the grid $(\mathbb{Z} / N)^{2}$ starting at the origin such that $\pi$ visits a balancing configuration at least every $N$ steps. We may assume that if $\pi$ visits a balancing configuration for the first time at time $n_{1}$, say, the partial sums up to that moment are all in $[0, N]$. Call this first-visited balancing configuration $W_{1}$. ( It is possible that $\pi_{n}$ is contained in the intersection of two balancing configurations in which case we just make a choice and call one of these $W_{1}$.) Let $m_{1}$ be the first time $m$ after $n_{1}$ for which $\pi_{m} \notin W_{1}$. Define $n_{2}$ as $\min \left\{n \geq m_{1}: \pi_{n}\right.$ visits a balancing configuration $\}$. Note that it is possible that $n_{2}=m_{1}$. The balancing configuration visited at time $n_{2}$ is denoted $W_{2}$, where it is again possible that we have a choice. Define $n_{k}, m_{k}$ and $W_{k}$ for $k=1,2, \ldots$ inductively in this fashion. We will now construct a just-visiting path $\pi^{\prime} . \pi^{\prime}$ will be constructed from $\pi$ by adding loops of repeating vertices each time $\pi$ visits a balancing configuration. More precisely, $\pi^{\prime}$ follows $\pi$ up to time $n_{1}$. Consider the sum of the labels along $\pi$ until $\pi$ visits the next balancing configuration $W_{2}$, that is, $S_{k}^{\pi}-S_{n_{1}}^{\pi}$, for $k=n_{1}+1, \ldots, n_{2}$. These numbers are uniformly bounded by $N$ in absolute value. We construct the next part of $\pi^{\prime}$ as follows. Pass around in the balancing configuration $W_{1}$ until you have reached a sum which guarantees that when we after that travel to $W_{2}$ along $\pi$ the partial sums will never be smaller than 0 or larger than $N+1$. When we are in $W_{2}$ we repeat this process. 
Proof of Theorem 4(b) We consider two possibilities:

(i) $\liminf _{N \rightarrow \infty} 2 N^{2}\left(1-q_{N}\right)^{2}=\infty$,

(ii) $\limsup \sup _{N \rightarrow \infty} 2 N^{2}\left(1-q_{N}\right)^{2}<\infty$.

For case (i) we take a grid square $S$, and we simply note that the probability that $S$ contains a balancing configuration converges to 1 as $N$ tends to infinity. Thus, with positive probability, there will be an infinite path of grid squares containing balancing configurations for $N$ sufficiently large. Hence the diamonds of the reduced diamond process must percolate, and in the same way as in the proof of Lemma 5.7 we can move around between balancing configurations to find our required path.

In case (ii) we can find $\lambda_{u p}, \lambda_{\text {down }}$ such that for all large $N$,

$$
\lambda_{c}<\lambda_{\text {down }}<2 N^{2}\left(1-q_{N-1}\right)^{2} q_{N-1}^{2}<\lambda_{\text {up }}<\infty .
$$

We can then define $\lambda_{N}$ as $2 N^{2}\left(1-q_{N-1}\right)^{2} q_{N-1}^{2}$ and apply Lemma 5.7 to this series of $\lambda_{N}$ s, which immediately gives the result.

Proof of Corollary 5 For this corollary we will show that each of the following three cases leads to a contradiction:

(i) $\liminf _{p \rightarrow 1} 2(1-p)^{2} I(p)^{2}<\lambda_{c}$,

(ii) $\lambda_{c}<\limsup _{p \rightarrow 1} 2(1-p)^{2} I(p)^{2}<\infty$,

(iii) $\limsup \sup _{p \rightarrow 1} 2(1-p)^{2} I(p)^{2}=\infty$.

In case (i) we can choose a sequence $p_{1}^{\circ}, p_{2}^{\circ}, \ldots$, such that $\lim _{i \rightarrow \infty} p_{i}^{\circ}=1$ and such that $\limsup _{i \rightarrow \infty} 2\left(1-p_{i}^{\circ}\right)^{2} I\left(p_{i}^{\circ}\right)^{2}<\lambda_{c}$. Now as $\lim _{i \rightarrow \infty} I\left(p_{i}^{\circ}\right)=\infty$ the set $\left\{I\left(p_{i}^{\circ}\right) ; i=1,2, \ldots\right\}$ must be infinite. This means that we can next define a new sequence, $p_{1}^{\bullet}, p_{2}^{\bullet}, \ldots$, which is a 'more regular' version of $\left\{p_{1}^{\circ}, p_{2}^{\circ}, \ldots\right\}$, in such a way that we can apply Theorem 4(a). So, if 
$\left\{p_{i}^{\circ}: I\left(p_{i}^{\circ}\right)=N\right\}$ is non-empty, then we choose $p_{N}^{\bullet}$ to be any element of this set. If it is empty, we set $p_{N}^{\bullet}$ equal to 1.

Then $\limsup _{N \rightarrow \infty} 2\left(1-p_{N+1}^{\bullet}\right)^{2} N^{2}<\lambda_{c}$, so by Theorem 4(a) we can find $N^{\prime}$ such that for all $N>N^{\prime}, \mathbb{P} p_{N^{-}}^{\bullet}$ almost surely, there are no paths with partial sums bounded in any interval of length $N$. However, from the construction we have that $I\left(p_{N}^{\bullet}\right)=N$ for infinitely many $N$, which contradicts the definition of $I$.

In case (ii) we proceed similarly but this time we use Theorem 4(b). Now we can choose a sequence $p_{1}^{\dagger}, p_{2}^{\dagger}, \ldots$, such that $\lim _{i \rightarrow \infty} p_{i}^{\dagger}=1$, $\limsup _{i \rightarrow \infty} 2\left(1-p_{i}^{\dagger}\right)^{2} I\left(p_{i}^{\dagger}\right)^{2}<\infty$ and $\liminf _{i \rightarrow \infty} 2\left(1-p_{i}^{\dagger}\right)^{2} I\left(p_{i}^{\dagger}\right)^{2}>\lambda_{c}$. Again we define a new version of this sequence. If the set $\left\{p_{i}^{\dagger}: I\left(p_{i}^{\dagger}\right)=N\right\}$ is non-empty, then we define $p_{N}^{\ddagger}$ to be any element of this set. If the set is empty, we set $p_{N}^{\ddagger}$ equal to $1-\sqrt{\frac{\lambda_{c}+1}{2 N^{2}}}$. Here the term $1-\sqrt{\frac{\lambda_{c}+1}{2 N^{2}}}$ is chosen to make $2\left(1-p_{N}^{\ddagger}\right)^{2} N^{2}=\lambda_{c}+1>\lambda_{c}$.

Next note that

$$
\limsup _{N \rightarrow \infty} 2\left(1-p_{N+1}^{\ddagger}\right)^{2}(N-1)^{2}<\infty
$$

and that

$$
\liminf _{N \rightarrow \infty} 2\left(1-p_{N+1}^{\ddagger}\right)^{2}(N-1)^{2}>\lambda_{c} .
$$

Thus by Theorem 4(b) there exists $N^{\prime \prime}$ such that for all $N>N^{\prime \prime}$ there are, with positive probability, paths with sums bounded in an interval of length $N-1$. Thus, by its minimality, $I\left(p_{N}^{\ddagger}\right)$ must be less than or equal to $N-1$, for $N>N^{\prime \prime}$. However we know that $I\left(p_{N}^{\ddagger}\right)=N$, which gives the required contradiction.

Case (iii) is proved in the same way as case (ii), by considering in this case a sequence of $p$ s for which $2(1-p)^{2} I(p)^{2}$ tends to infinity in the limit. The only necessary modifications are to define $p_{N}^{\ddagger}$ as an arbitrary constant 
between $1 / 2$ and 1 for cases where $\left\{p_{i}^{\dagger}: I\left(p_{i}^{\dagger}\right)=N\right\}$ is empty, and to use the other condition in Theorem 4(b).

\section{Proofs of Theorem 6 and Corollary 7}

Proof of Theorem 6 We give a proof by contradiction, so we first assume that there is a value of $p>1 / 2$ such that both,

$$
\mathbb{P}_{p}\left(\exists \text { a path } \pi \text { for which } S_{n}^{\pi} \text { does not converge to }+\infty\right)>0,
$$

and,

$$
\mathbb{P}_{p}\left(\exists \text { a path } \pi \text { with } S_{n}^{\pi}=0 \text { i.o. }\right)=0 .
$$

Observe that for $p>1 / 2$, we can take, for example, the path given by the positive part of the $x$-axis to give an infinite self-avoiding path $\pi^{u}$ such that, with probability 1 ,

$$
\lim _{n \rightarrow \infty} S_{n}^{\pi^{u}}=\infty
$$

In the proof of Lemma 4.1 we showed that (10) implies that for all $z \in \mathbb{Z}$,

$$
\mathbb{P}_{p}\left(\exists \text { a path } \pi \text { with } S_{n}^{\pi}=z \text { i.o. }\right)=0 .
$$

From this and (9) it follows that there exists with positive probability a path $\pi^{d}$ with

$$
\lim _{n \rightarrow \infty} S_{n}^{\pi^{d}}=-\infty
$$

We next construct from these two paths a new self-avoiding path with partial sums that return to zero infinitely often, by moving between the two paths in a way that ensures that the resulting path does not cross itself. This will then contradict (10). 


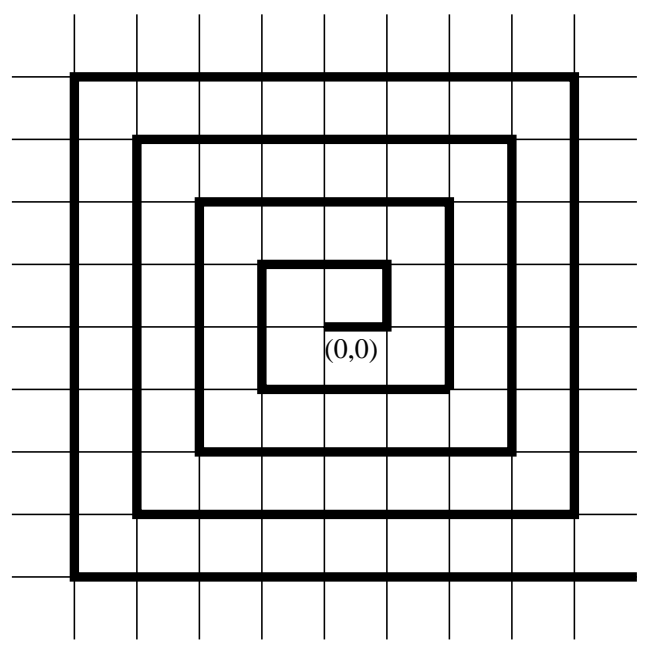

Figure 4: The spiral $S$

We will speak of a point on a self-avoiding path as being before another (or as an earlier point) if it is nearer to the origin, measuring along the path. Points that are after others (or later points) are defined analogously.

For the construction we use the spiral $S$, shown in Figure 4, which covers all points in the square lattice, and is self-avoiding. We define a spiral circuit from a point, $\alpha$, to be that sub-path of $S$, starting at $\alpha$, which passes around the origin until at Euclidean distance $\sqrt{2}$ from $\alpha$, see Figure 5 . We note two things:

(a) There are an infinite number of points on any self-avoiding path from the origin which have the property that no earlier points on the path intersect later points of the spiral.

(b) There exists a last intersection of a self-avoiding path and any spiral circuit, in the ordering given by the path.

We construct our new path $\pi^{n}$ as follows, after starting by following (i.e. copying the steps of) $\pi^{d}$ for a little while: 


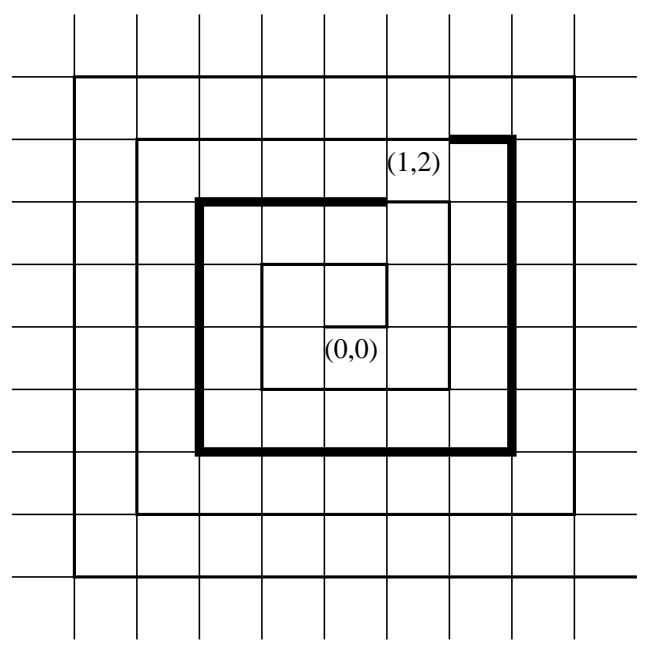

Figure 5: The spiral circuit from the point $(1,2)$

1. Follow $\pi^{d}$ until a point when both the partial sum of $\pi^{n}$ is below zero and none of the points on $S$ after this point are already in $\pi^{n}$. This is possible by (a), and the fact that the partial sums of $\pi^{d}$ are only above or at any value for a finite number of steps.

2. Find the last intersection between $\pi^{u}$ and the spiral circuit from the point where we currently are, and move along $S$ to this point. This is possible by (b). By the previous step, $\pi^{n}$ avoids any earlier parts of itself (which were strictly inside the spiral circuit).

3. Follow $\pi^{u}$ until a point when both the partial sum of $\pi^{n}$ is above zero and none of the points on $S$ after this point are already in $\pi^{n}$. Note that $\pi^{u}$ will never return to earlier points in $\pi^{n}$, by the previous step.

4. Find the last intersection between $\pi^{d}$ and the spiral circuit from the point where we currently are, and move along $S$ to this point.

5. Repeat. 
Notice that it is always possible to keep passing from path to path in this manner, and thus the partial sums of $\pi^{n}$ are zero infinitely often. We have a contradiction, and so our result.

Proof of Corollary 7 The existence of paths with partial sums which return to zero infinitely often is clearly a tail event and so has probability 1 or 0 , by Kolmogorov's $0-1$ law.

Assign to each vertex $v \in \mathbb{Z}^{2}$ a uniform $[0,1]$ random variable, $U_{v}$. We can use these variables to couple realisations at all parameter values together such that if $\left\{\begin{array}{l}U_{v} \geq 1-p \text { then } \quad X_{v}=1, \\ U_{v}<1-p \text { then } \quad X_{v}=-1 .\end{array}\right.$

Theorem 6 states that if with positive probability there is a path with partial sums not converging to $+\infty$, then there exists with positive probability, and hence with probability one, a path with partial sums that return to zero infinitely often. On the other hand, if all paths have partial sums converging to $+\infty$, then clearly there does not exist a path with partial sums that return to zero infinitely often. Hence we have a path with partial sums that return to zero infinitely often, with positive probability, if and only if there is a path, with positive probability, whose partial sums do not converge to $+\infty$. From the coupling described above we see immediately that the probability of the event that all paths have partial sums converging to $+\infty$ is monotone in $p$. It follows that the probability of having a path with partial sums that return to zero infinitely often is also monotone in $p$.

Acknowledgements We thank Deepak Dhar and Jeffrey Steif for various suggestions and fruitful discussions. 


\section{References}

[1] Sven Erick Alm. Upper boundes for the connective constant of selfavoiding walks. Combinatorics, Probability and Computing, 2(2):115$136,1993$.

[2] Martin J. Appel and John C. Wierman. Infinite AB clusters exist on the triangular lattice. Journal of Physics A: Mathematical and General, $20: 2533-2537,1987$.

[3] Martin J. Appel and John C. Wierman. On the absence of infinite AB clusters in bipartite graphs. Journal of Physics A: Mathematical and General, 20:2527-2531, 1987.

[4] A.D. Barbour, Lars Holst, and Svante Janson. Poisson Approximation. Oxford Science Publications, 1992.

[5] Itai Benjamini and Yuval Peres. Markov chains indexed by trees. Annals of Probability, 22(1), 1994.

[6] D.J. Daley and D. Vere-Jones. An Introduction to the Theory of Point Processes. Springer, 1980.

[7] Kenneth J. Falconer. Fractal Geometry: Mathematical Foundations and Applications. Wiley, 1990.

[8] G. R. Grimmett and D.R. Stirzaker. Probability and Random Processes. Oxford University Press, second edition, 1992.

[9] Geoffrey R. Grimmett. Percolation. Springer, second edition, 1999.

[10] Harry Kesten. Percolation for Mathematicians. Birkhauser, 1980. 
[11] Thomas M. Liggett. Survival of discrete time growth models, with applications to oriented percolation. Annals of Applied Probability, $5(3): 613-636,1995$.

[12] Tomasz Luczak and John C. Wierman. Counterexamples in AB percolation. Journal of Physics A: Mathematical and General, 22:185-191, 1989 .

[13] Ronald Meester and Rahul Roy. Continuum Percolation. Cambridge University Press, 1996. 